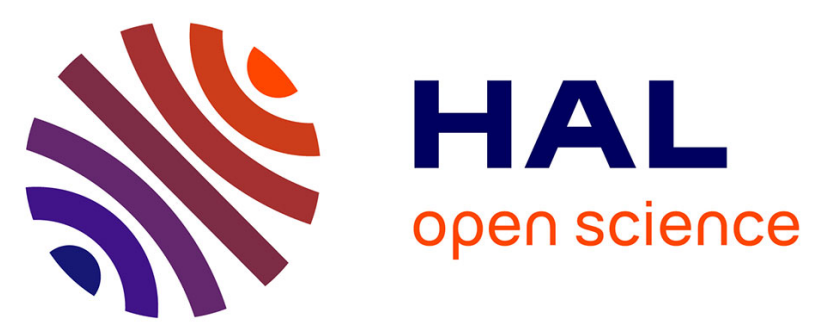

\title{
Quaternion Zernike moments and their invariants for color image analysis and object recognition
}

Beijing Chen, Huazhong Shu, Hui Zhang, Gang Chen, Christine Toumoulin, Jean-Louis Dillenseger, Limin M. Luo

\section{- To cite this version:}

Beijing Chen, Huazhong Shu, Hui Zhang, Gang Chen, Christine Toumoulin, et al.. Quaternion Zernike moments and their invariants for color image analysis and object recognition. Signal Processing, 2012, 92 (2), pp.308-318. 10.1016/j.sigpro.2011.07.018 . inserm-00639532

\section{HAL Id: inserm-00639532 https://www.hal.inserm.fr/inserm-00639532}

Submitted on 9 Nov 2011

HAL is a multi-disciplinary open access archive for the deposit and dissemination of scientific research documents, whether they are published or not. The documents may come from teaching and research institutions in France or abroad, or from public or private research centers.
L'archive ouverte pluridisciplinaire HAL, est destinée au dépôt et à la diffusion de documents scientifiques de niveau recherche, publiés ou non, émanant des établissements d'enseignement et de recherche français ou étrangers, des laboratoires publics ou privés. 


\title{
Quaternion Zernike moments and their invariants for color image analysis and object recognition
}

\author{
B.J. Chen ${ }^{\mathrm{a}, \mathrm{de}}$, H.Z. Shu ${ }^{\mathrm{a}, \mathrm{d}, *}$, H. Zhang ${ }^{\mathrm{a}}$, G. Chen ${ }^{\mathrm{a}}$, C. Toumoulin ${ }^{\mathrm{b}, \mathrm{c}, \mathrm{d}}$, J.L. Dillenseger ${ }^{\mathrm{b}, \mathrm{c}, \mathrm{d}}$, L.M. Luo ${ }^{\mathrm{a}, \mathrm{d}}$ \\ ${ }^{a}$ Laboratory of Image Science and Technology, Southeast University, Nangjing 210096, China \\ ${ }^{b}$ INSERM, U642, Rennes, F-35000, France \\ ${ }^{c}$ Université de Rennes 1, LTSI, Rennes, F-35000, France \\ ${ }^{d}$ Centre de Recherche en Information Biomédicale Sino-Français (CRIBs) \\ ${ }^{e}$ School of Electronic and Information Engineering, Ningbo University of Technology, Ningbo 315016, China
}

\begin{abstract}
Moments and moment invariants have become a powerful tool in pattern recognition and image analysis. Conventional methods to deal with color images are based on RGB decomposition or graying, which may lose some significant color information. In this paper, by using the algebra of quaternions, we introduce the quaternion Zernike moments (QZMs) to deal with the color images in a holistic manner. It is shown that the QZMs can be obtained from the conventional Zernike moments of each channel. We also provide the theoretical framework to construct a set of combined invariants with respect to rotation, scaling and translation (RST) transformation. Experimental results are provided to illustrate the efficiency of the proposed descriptors.
\end{abstract}

Keywords: Color image; Quaternion; Zernike moment; Moment invariant.

\section{Introduction}

Moment invariants have been extensively used in pattern recognition [1], [2], scene matching [3] and object classification [4] owing to their image description capability and invariance property. However, they are mainly used to deal with the binary or gray-scale images. With the development of inexpensive digital camera, nowadays almost all images acquired are chromatic. Generally speaking, there are two approaches that are often used for color image processing. The first one consists of transforming the color image into gray-scale one, which may lose some significant color information. For example, if we use the average of three channels for graying the color image, it is impossible to identify the objects having the same shape but different color (red, green, blue). The second one decomposes the color image into three channels, and then calculates the moment invariants of these three channels separately [5]-[8]. Among the latter, Mindru et al. [5], [6] proposed the generalized color moments for color images, whose integral function is the product of the powers of the pixel coordinates and those of the intensities for one or more color channels. Based on these moments, they constructed a set of invariants to geometric transformation and photometric changes. Suk and Flusser [7] derived a set of affine geometric invariants for color images, which are based on the product of moments defined for different channels. Specially, they introduced the notion of common centroid for defining the central moments in order to achieve the translation invariance. Both methods are the generalization of conventional geometric moments, the difference between them is that the computation of Mindru's moments may refer to two or three color channels, and that of Suk's moments only relates to one channel. Since the kernel functions of geometric moments are not orthogonal, this may lead to information redundancy and low noise robustness.

The orthogonal Zernike polynomials were first introduced by Zernike in 1934 [9], Teague used them to define the orthogonal Zernike moments [10]. Since then, the Zernike moments have been applied to a number of computer vision problems [1], [11]-[18] because they have overall better performance than other moments [19]-[21]. The purpose of this paper is to extend the conventional orthogonal Zernike moments to color image in a holistic manner. To that end, we will use the algebra of quaternions.

In recent years, quaternions have been utilized more and more in color image processing. Let $f(x, y)$ be an RGB image, it can be represented by encoding three channels as a pure quaternion as follows

$$
f(x, y)=f_{R}(x, y) \boldsymbol{i}+f_{G}(x, y) \boldsymbol{j}+f_{B}(x, y) \boldsymbol{k},
$$

where $f_{R}(x, y), f_{G}(x, y)$ and $f_{B}(x, y)$ represent respectively the red, green and blue channel of the color images, and $\boldsymbol{i}, \boldsymbol{j}$ and $\boldsymbol{k}$ are the complex operators. The main advantage of the use of quaternion-type representation is that a color image can be

\footnotetext{
* Corresponding author. Tel.: +00-86-25-83 7942 49; fax: +00-86-25-83 7926 98; E-mail address: shu.list@ seu.edu.cn.
} 
treated as a vector field [22]. The algebra of quaternions has been exploited in color image processing by Ell and Sangwine [22]-[24]. In 1992, Ell [23] introduced the quaternion Fourier transforms (QFTs) in his Ph. D dissertation. Sangwine [24] then applied them to color image. Since then, the QFTs have been successfully employed in color image registration [25], [26], watermarking [27], [28], motion estimation [29] and texture analysis [30]. Recently, the use of quaternion-based moment functions to color image has been investigated [31], [32]. We have introduced the notion of the quaternion Zernike moments (QZMs) and derived a set of invariants with respect to image translation and rotation [31]. By using the quaternion algebra, Guo et al. proposed the quaternion Fourier-Mellin moments (QFMMs) to deal with the color images and constructed a set of invariants to rotation, scale and translation (RST) transformation [32]. However, in both methods, the rotation invariance was achieved by taking the modulus of the quaternion moments, this may lead to two disadvantages: 1) the modulus loses the phase information which may be useful in some applications; 2) the modulus only provides one real-valued invariant.

In this paper, we propose a new approach to construct a set of QZM invariants with respect to RST transformation. The remaining of this paper is organized as follows. In Section 2, we first recall some preliminaries about the quaternions, and then present the definition of the QZMs. In Section 3, we derive a set the moment invariants with respect to RST transformation. Experimental results are provided in Section 4 to illustrate the performance of the proposed descriptors. Section 5 concludes the paper.

\section{Quaternion moments}

\subsection{Quaternion number}

Quaternions, a generalization of the complex numbers, were introduced by the mathematician Hamilton in 1843 [33]. A quaternion consists of one real part and three imaginary parts as follows

$$
q=a+b \boldsymbol{i}+c \boldsymbol{j}+d \boldsymbol{k} .
$$

where $a, b, c$ and $d$ are real numbers, and $\boldsymbol{i}, \boldsymbol{j}$ and $\boldsymbol{k}$ are three imaginary units obeying the following rules

$$
\begin{aligned}
& \boldsymbol{i}^{2}=\boldsymbol{j}^{2}=\boldsymbol{k}^{2}=-1, \\
& \boldsymbol{i} \boldsymbol{j}=-\boldsymbol{j} \boldsymbol{i}=\boldsymbol{k}, \boldsymbol{j} \boldsymbol{k}=-\boldsymbol{k} \boldsymbol{j}=\boldsymbol{i}, \boldsymbol{k i}=-\boldsymbol{i} \boldsymbol{k}=\boldsymbol{j} .
\end{aligned}
$$

The conjugate and modulus of a quaternion are respectively defined by

$$
\begin{aligned}
q^{*} & =a-b \boldsymbol{i}-c \boldsymbol{j}-d \boldsymbol{k}, \\
|q| & =\sqrt{a^{2}+b^{2}+c^{2}+d^{2}} .
\end{aligned}
$$

\subsection{Quaternion Zernike moments}

Let $f(r, \theta)$ be an RGB image defined in polar coordinates, we define the right-side QZMs of order $n$ with repetition $m$ as [31]

$$
Z_{n, m}^{R}(f)=\frac{n+1}{\pi} \int_{0}^{1} \int_{0}^{2 \pi} R_{n, m}(r) f(r, \theta) e^{-\mu m \theta} r d \theta d r,|m| \leq n \text { and } n-|m| \text { being even, }
$$

where $\boldsymbol{\mu}$ is a unit pure quaternion chosen as $\boldsymbol{\mu}=(\boldsymbol{i}+\boldsymbol{j}+\boldsymbol{k}) / \sqrt{3}$ in this paper, $R_{n, m}(r)$ is the real-valued radial polynomial given by

$$
R_{n, m}(r)=\sum_{k=0}^{(n-|m|) / 2} \frac{(-1)^{k}(n-k) !}{k !\left(\frac{n+|m|}{2}-k\right) !\left(\frac{n-|m|}{2}-k\right) !} r^{n-2 k} .
$$

Since the radial Zernike polynomials are orthogonal, the color image $f(r, \theta)$ can be approximatively reconstructed from a finite number $M$ of QZMs as follows

$$
f(r, \theta) \approx \sum_{n=0}^{M} \sum_{m=-n}^{n} Z_{n, m}^{R}(f) R_{n, m}(r) e^{\boldsymbol{\mu} m \theta} .
$$

Because the Zernike moments are defined in polar coordinates $(r, \theta)$ with $|r| \leq 1$, the computation of Zernike moments requires a linear transformation of the image coordinates to a suitable domain inside a unit circle. Here we use the mapping transformation proposed by Chong et al. [34]. Based on this transformation, we have the following discrete approximation of (7):

$$
Z_{n, m}^{R}(f)=\frac{n+1}{\pi(N-1)^{2}} \sum_{x=1}^{N} \sum_{y=1}^{N} R_{n, m}\left(r_{x, y}\right) f(x, y) e^{-\mu m \theta_{x, y}} .
$$


where $N$ is the number of pixels along each axis of the image, the mapping transformation to the interior of the unit circle is given by

$$
r_{x, y}=\sqrt{\left(c_{1} x+c_{2}\right)^{2}+\left(c_{1} y+c_{2}\right)^{2}}, \quad \theta_{x, y}=\tan ^{-1}\left(\frac{c_{1} y+c_{2}}{c_{1} x+c_{2}}\right),
$$

with $c_{1}=\frac{\sqrt{2}}{N-1}, c_{2}=-\frac{1}{\sqrt{2}}$.

It can be seen from (4) that the multiplication of quaternions is not commutative, so we also define the left-side QZMs of order $n$ with repetition $m$ as

$$
Z_{n, m}^{L}(f)=\frac{n+1}{\pi} \int_{0}^{1} \int_{0}^{2 \pi} R_{n, m}(r) e^{-\mu m \theta} f(r, \theta) r d \theta d r,
$$

The corresponding inverse transform is

$$
f(r, \theta) \approx \sum_{n=0}^{M} \sum_{m=-n}^{n} e^{\boldsymbol{\mu} m \theta} Z_{n, m}^{L}(f) R_{n, m}(r) .
$$

2.3. Relationship between the quaternion Zernike moments and the conventional Zernike moments for single channel of the $R G B$ image

Substituting (1) into (7) and using (3) and (4), we obtain

$$
\begin{aligned}
& Z_{n, m}^{R}(f)=\frac{n+1}{\pi} \int_{0}^{1} \int_{0}^{2 \pi} R_{n, m}(r)\left[f_{R}(r, \theta) \boldsymbol{i}+f_{G}(r, \theta) \boldsymbol{j}+f_{B}(r, \theta) \boldsymbol{k}\right] e^{-\boldsymbol{\mu} n \theta} r d \theta d r \\
& =i \frac{n+1}{\pi} \int_{0}^{1} \int_{0}^{2 \pi} R_{n, m}(r) f_{R}(r, \theta) e^{-\mu n \theta} r d \theta d r+j \frac{n+1}{\pi} \int_{0}^{1} \int_{0}^{2 \pi} R_{n, m}(r) f_{G}(r, \theta) e^{-\mu n \theta} r d \theta d r \\
& +\boldsymbol{k} \frac{n+1}{\pi} \int_{0}^{1} \int_{0}^{2 \pi} R_{n, m}(r) f_{B}(r, \theta) e^{-\mu m \theta} r d \theta d r \\
& =i \frac{n+1}{\pi} \int_{0}^{1} \int_{0}^{2 \pi} R_{n, m}(r) f_{R}(r, \theta)(\cos m \theta-\mu \sin m \theta) r d \theta d r+j \frac{n+1}{\pi} \int_{0}^{1} \int_{0}^{2 \pi} R_{n, m}(r) f_{G}(r, \theta)(\cos m \theta-\mu \sin m \theta) r d \theta d r \\
& +\boldsymbol{k} \frac{n+1}{\pi} \int_{0}^{1} \int_{0}^{2 \pi} R_{n, m}(r) f_{B}(r, \theta)(\cos m \theta-\mu \sin m \theta) r d \theta d r \\
& =i\left[\frac{n+1}{\pi} \int_{0}^{1} \int_{0}^{2 \pi} R_{n, m}(r) f_{R}(r, \theta) \cos (m \theta) r d \theta d r-\mu \frac{n+1}{\pi} \int_{0}^{1} \int_{0}^{2 \pi} R_{n, m}(r) f_{R}(r, \theta) \sin (m \theta) r d \theta d r\right] \\
& +j\left[\frac{n+1}{\pi} \int_{0}^{1} \int_{0}^{2 \pi} R_{n, m}(r) f_{G}(r, \theta) \cos (m \theta) r d \theta d r-\mu \frac{n+1}{\pi} \int_{0}^{1} \int_{0}^{2 \pi} R_{n, m}(r) f_{G}(r, \theta) \sin (m \theta) r d \theta d r\right] \\
& +\boldsymbol{k}\left[\frac{n+1}{\pi} \int_{0}^{1} \int_{0}^{2 \pi} R_{n, m}(r) f_{B}(r, \theta) \cos (m \theta) r d \theta d r-\mu \frac{n+1}{\pi} \int_{0}^{1} \int_{0}^{2 \pi} R_{n, m}(r) f_{B}(r, \theta) \sin (m \theta) r d \theta d r\right] \\
& =\boldsymbol{i}\left[\operatorname{Re}\left(Z_{n, m}\left(f_{R}\right)\right)+\frac{(\boldsymbol{i}+\boldsymbol{j}+\boldsymbol{k})}{\sqrt{3}} \operatorname{Im}\left(Z_{n, m}\left(f_{R}\right)\right)\right]+\boldsymbol{j}\left[\operatorname{Re}\left(Z_{n, m}\left(f_{G}\right)\right)+\frac{(\boldsymbol{i}+\boldsymbol{j}+\boldsymbol{k})}{\sqrt{3}} \operatorname{Im}\left(Z_{n, m}\left(f_{G}\right)\right)\right] \\
& +\boldsymbol{k}\left[\operatorname{Re}\left(Z_{n, m}\left(f_{B}\right)\right)+\frac{(\boldsymbol{i}+\boldsymbol{j}+\boldsymbol{k})}{\sqrt{3}} \operatorname{Im}\left(Z_{n, m}\left(f_{B}\right)\right)\right] \\
& =A_{n, m}^{R}+\boldsymbol{i} B_{n, m}^{R}+j C_{n, m}^{R}+k D_{n, m}^{R} \text {, }
\end{aligned}
$$

where 


$$
\begin{aligned}
& A_{n, m}^{R}=-\frac{1}{\sqrt{3}}\left[\operatorname{Im}\left(Z_{n, m}\left(f_{R}\right)\right)+\operatorname{Im}\left(Z_{n, m}\left(f_{G}\right)\right)+\operatorname{Im}\left(Z_{n, m}\left(f_{B}\right)\right)\right], \\
& B_{n, m}^{R}=\operatorname{Re}\left(Z_{n, m}\left(f_{R}\right)\right)+\frac{1}{\sqrt{3}}\left[\operatorname{Im}\left(Z_{n, m}\left(f_{G}\right)\right)-\operatorname{Im}\left(Z_{n, m}\left(f_{B}\right)\right)\right], \\
& C_{n, m}^{R}=\operatorname{Re}\left(Z_{n, m}\left(f_{G}\right)\right)+\frac{1}{\sqrt{3}}\left[\operatorname{Im}\left(Z_{n, m}\left(f_{B}\right)\right)-\operatorname{Im}\left(Z_{n, m}\left(f_{R}\right)\right)\right], \\
& D_{n, m}^{R}=\operatorname{Re}\left(Z_{n, m}\left(f_{B}\right)\right)+\frac{1}{\sqrt{3}}\left[\operatorname{Im}\left(Z_{n, m}\left(f_{R}\right)\right)-\operatorname{Im}\left(Z_{n, m}\left(f_{G}\right)\right)\right] .
\end{aligned}
$$

Here $Z_{n, m}\left(f_{R}\right), Z_{n, m}\left(f_{G}\right)$ and $Z_{n, m}\left(f_{B}\right)$ are respectively the conventional Zernike moments for the red channel, green channel and blue channel, $\operatorname{Re}(x)$ represents the real part of conventional complex number $x$, and $\operatorname{Im}(x)$ the imaginary part, that is, $\operatorname{Re}(a+$ $b \boldsymbol{i})=a, \operatorname{Im}(a+b \boldsymbol{i})=b$.

Equation (14) shows that the QZMs can be obtained from the conventional Zernike moments for single channel. Similarly, we have the following relationship for the left-side QZMs

$$
Z_{n, m}^{L}(f)=A_{n, m}^{L}+i B_{n, m}^{L}+j C_{n, m}^{L}+k D_{n, m}^{L},
$$

where

$$
\begin{aligned}
& A_{n, m}^{L}=-\frac{1}{\sqrt{3}}\left[\operatorname{Im}\left(Z_{n, m}\left(f_{R}\right)\right)+\operatorname{Im}\left(Z_{n, m}\left(f_{G}\right)\right)+\operatorname{Im}\left(Z_{n, m}\left(f_{B}\right)\right)\right], \\
& B_{n, m}^{L}=\operatorname{Re}\left(Z_{n, m}\left(f_{R}\right)\right)+\frac{1}{\sqrt{3}}\left[\operatorname{Im}\left(Z_{n, m}\left(f_{B}\right)\right)-\operatorname{Im}\left(Z_{n, m}\left(f_{G}\right)\right)\right], \\
& C_{n, m}^{L}=\operatorname{Re}\left(Z_{n, m}\left(f_{G}\right)\right)+\frac{1}{\sqrt{3}}\left[\operatorname{Im}\left(Z_{n, m}\left(f_{R}\right)\right)-\operatorname{Im}\left(Z_{n, m}\left(f_{B}\right)\right)\right], \\
& D_{n, m}^{L}=\operatorname{Re}\left(Z_{n, m}\left(f_{B}\right)\right)+\frac{1}{\sqrt{3}}\left[\operatorname{Im}\left(Z_{n, m}\left(f_{G}\right)\right)-\operatorname{Im}\left(Z_{n, m}\left(f_{R}\right)\right)\right] .
\end{aligned}
$$

Comparing (14), (15) with (16), (17), we have

$$
Z_{n, m}^{L}(f)=-\left(Z_{n,-m}^{R}(f)\right)^{*}
$$

\section{RST invariants of quaternion Zernike moments}

In our previous work [31], we have derived the translation and rotation invariants of QZMs. The rotation invariance was achieved by taking the modulus of QZMs, which leads to some drawbacks as noted in the Introduction. Here, we propose a new approach to construct the rotation invariants. We will also discuss the way to derive the scaling invariants. Before that, we give a brief description of translation invariants proposed in [31].

\subsection{Translation invariants [31]}

Suk and Flusser [7] have defined the common centroid $\left(x_{c}, y_{c}\right)$ of all three channels as follows

$$
\begin{aligned}
& x_{c}=\left(m_{1,0}\left(f_{R}\right)+m_{1,0}\left(f_{G}\right)+m_{1,0}\left(f_{B}\right)\right) / m_{0,0}, \\
& y_{c}=\left(m_{0,1}\left(f_{R}\right)+m_{0,1}\left(f_{G}\right)+m_{0,1}\left(f_{B}\right)\right) / m_{0,0}, \\
& m_{0,0}=m_{0,0}\left(f_{R}\right)+m_{0,0}\left(f_{G}\right)+m_{0,0}\left(f_{B}\right),
\end{aligned}
$$

where $m_{0,0}\left(f_{R}\right), m_{1,0}\left(f_{R}\right)$ and $m_{0,1}\left(f_{R}\right)$ are respectively the zero-order and first-order geometric moment for $R$ channel, $m_{0,0}\left(f_{G}\right)$, $m_{1,0}\left(f_{G}\right)$ and $m_{0,1}\left(f_{G}\right)$ for $G$ channel, and $m_{0,0}\left(f_{B}\right), m_{1,0}\left(f_{B}\right)$ and $m_{0,1}\left(f_{B}\right)$ for $B$ channel.

Let the origin of the coordinate system be located at $\left(x_{c}, y_{c}\right)$, the central QZMs, which are invariant to image translation, can be obtained as follows

$$
\bar{Z}_{n, m}^{R}(f)=\frac{n+1}{\pi} \int_{0}^{1} \int_{0}^{2 \pi} R_{n, m}(\bar{r}) f(\bar{r}, \bar{\theta}) e^{-\mu m \bar{\theta}} \bar{r} d \bar{\theta} d \bar{r},
$$

where $(\bar{r}, \bar{\theta})$ is the image pixel coordinate representation in polar form with the mapping transformation (11) by locating the origin at $\left(x_{c}, y_{c}\right)$. 


\subsection{Rotation invariants}

Let $f^{\prime}$ be the rotated version of $f$, i.e., $f^{\prime}(r, \theta)=f(r, \theta-\alpha)$, where $\alpha$ denotes the rotation angle, then we have

$$
\begin{aligned}
Z_{n, m}^{R}\left(f^{\prime}\right) & =\frac{n+1}{\pi} \int_{0}^{1} \int_{0}^{2 \pi} R_{n, m}(r) f^{\prime}(r, \theta) e^{-\mu m \theta} r d \theta d r \\
= & \frac{n+1}{\pi} \int_{0}^{1} \int_{0}^{2 \pi} R_{n, m}(r) f(r, \theta-\alpha) e^{-\mu m \theta} r d \theta d r \\
= & \frac{n+1}{\pi} \int_{0}^{1} \int_{0}^{2 \pi} R_{n, m}(r) f(r, \theta) e^{-\mu m(\theta+\alpha)} r d \theta d r \\
= & \frac{n+1}{\pi} \int_{0}^{1} \int_{0}^{2 \pi} R_{n, m}(r) f(r, \theta) e^{-\mu m \theta} r d \theta d r \cdot e^{-\mu m \alpha} \\
= & Z_{n, m}^{R}(f) e^{-\mu m \alpha} .
\end{aligned}
$$

Equation (22) shows that the modulus of $Z_{n, m}^{R}$ is invariant to rotation, which is the method proposed in [31] to achieve the rotation invariance. However, such a process loses the phase information which may be useful in some applications. Moreover, $\left|Z_{n, m}^{R}\right|$ provides only one real-valued invariant. To surmount these shortcomings, we propose here a new way to construct a set of quaternion-valued rotation invariants.

By proceeding in a similar way as for (21), we can obtain the following relationship for the left-side QZMs

$$
Z_{n, m}^{L}\left(f^{\prime}\right)=e^{-\mu m \alpha} Z_{n, m}^{L}(f) \text {. }
$$

Theorem 1. Let

$$
\phi_{n, k}^{m}(f)=Z_{n, m}^{R}(f) Z_{k,-m}^{L}(f)=-Z_{n, m}^{R}(f)\left(Z_{k, m}^{R}(f)\right)^{*},|m| \leq n,|m| \leq k, n-|m| \text { and } k-|m| \text { being even. }
$$

Then, $\phi_{n, k}^{m}(f)$ is invariant to image rotation for any integer $m$ and non-negative integer $n$ and $k$.

Proof. Let $f^{\prime}$ be the rotated image of $f$ with rotation angle $\alpha$, using (21) and (22), we have

$$
\begin{aligned}
\phi_{n, k}^{m}\left(f^{\prime}\right) & =Z_{n, m}^{R}\left(f^{\prime}\right) Z_{k,-m}^{L}\left(f^{\prime}\right) \\
& =Z_{n, m}^{R}(f) e^{-\mu m \alpha} e^{\mu m \alpha} Z_{k,-m}^{L}(f) \\
& =Z_{n, m}^{R}(f) Z_{k,-m}^{L}(f)=\phi_{n, k}^{m}(f)
\end{aligned}
$$

The proof has been completed.

Based on Theorem 1, when $k=n$, we have $\phi_{n, n}^{m}(f)=-\left|Z_{n, m}^{R}(f)\right|^{2}$. It means that the rotation invariants constructed by taking the modulus of $Z_{n, m}^{R}(f)$ correspond to a special case of Theorem 1. Note that each invariant $\phi_{n, k}^{m}(f)$ is a quaternion number, which includes four real-valued invariants (one real part and three imaginary parts) except $\phi_{n, n}^{m}(f)$.

\subsection{Scaling invariants and combined RST invariants}

In this subsection, we first derive a set of invariants with respect to image scale. Equation (8) shows that the radial polynomial $R_{n, m}(r)$ is symmetric with $m$, that is, $R_{n,-m}(r)=R_{n, m}(r)$. So, the case $m \geq 0$ is considered. Letting $n=m+2 l$ and using (8), (7) can be rewritten as

$$
\begin{aligned}
Z_{m+2 l, m}^{R}(f) & =\frac{m+2 l+1}{\pi} \int_{0}^{1} \int_{0}^{2 \pi}\left[\sum_{k=0}^{l}(-1)^{k} \frac{(m+2 l-k) !}{k !(m+l-k) !(l-k) !} r^{m+2(l-k)}\right] f(r, \theta) e^{-\mu m \theta} r d \theta d r \\
& =\frac{m+2 l+1}{\pi} \int_{0}^{1} \int_{0}^{2 \pi}\left[\sum_{k=0}^{l}(-1)^{l-k} \frac{(m+l+k) !}{k !(m+k) !(l-k) !} r^{m+2 k}\right] f(r, \theta) e^{-\mu n \theta} r d \theta d r \\
& =\sum_{k=0}^{l}(-1)^{l-k} \frac{m+2 l+1}{\pi} \frac{(m+l+k) !}{k !(m+k) !(l-k) !} \int_{0}^{12 \pi} \int_{0}^{2 \pi} r^{m+2 k} f(r, \theta) e^{-\mu m \theta} r d \theta d r \\
& =\sum_{k=0}^{l} c_{l, k}^{m} \psi_{m+2 k, m}^{R}(f),
\end{aligned}
$$

where

$$
\psi_{m+2 k, m}^{R}(f)=\int_{0}^{1} \int_{0}^{2 \pi} r^{m+2 k} f(r, \theta) e^{-\boldsymbol{\mu} m \theta} r d \theta d r
$$




$$
c_{l, k}^{m}=(-1)^{l-k} \frac{m+2 l+1}{\pi} \frac{(m+l+k) !}{k !(l-k) !(m+k) !} .
$$

From $(25), \psi_{m+2 l, m}^{R}(f)$ can also be expressed as a series of QZMs

$$
\psi_{m+2 l, m}^{R}(f)=\sum_{k=0}^{l} d_{l, k}^{m} Z_{m+2 k, m}^{R}(f)
$$

where $D_{l}^{m}=\left(d_{i, j}^{m}\right)$, with $0 \leq j \leq i \leq l$, is the inverse matrix of $C_{l}^{m}=\left(c_{i, j}^{m}\right)$. The elements of $D_{l}^{m}$ are given by [35]

$$
d_{i, j}^{m}=\frac{i !(m+i) ! \pi}{(i-j) !(m+i+j+1) !}, \quad 0 \leq j \leq i \leq l .
$$

Let $f^{\prime \prime}$ and $f$ be two images having the same content but scale $(\lambda)$, that is, $f^{\prime \prime}(r, \theta)=f(r / \lambda, \theta)$, using (25) and (28), the QZMs of the transformed image can be obtained as

$$
\begin{aligned}
Z_{m+2 l, m}^{R}\left(f^{\prime \prime}\right) & =\sum_{k=0}^{l} c_{l, k}^{m} \int_{0}^{1} \int_{0}^{2 \pi} r^{m+2 k} f(r / \lambda, \theta) e^{-\boldsymbol{\mu} m \theta} r d \theta d r \\
& =\sum_{k=0}^{l} c_{l, k}^{m} \lambda^{2} \int_{0}^{1} \int_{0}^{2 \pi}(\lambda r)^{m+2 k} f(r, \theta) e^{-\boldsymbol{\mu} m \theta} r d \theta d r \\
& =\sum_{k=0}^{l} \lambda^{m+2 k+2} c_{l, k}^{m} \psi_{m+2 k, m}^{R}(f) \\
& =\sum_{k=0}^{l} \sum_{t=0}^{k} \lambda^{m+2 k+2} c_{l, k}^{m} d_{k, t}^{m} Z_{m+2 t, m}^{R}(f) \\
& =\sum_{t=0}^{l} \sum_{k=t}^{l} \lambda^{m+2 k+2} c_{l, k}^{m} d_{k, t}^{m} Z_{m+2 t, m}^{R}(f) .
\end{aligned}
$$

Theorem 2. Let

$$
L_{m+2 l, m}^{R}(f)=\sum_{t=0}^{l} \sum_{k=t}^{l} \Gamma_{f}^{-(m+2 k+2)} c_{l, k}^{m} d_{k, t}^{m} Z_{m+2 t, m}^{R}(f),
$$

with $\Gamma_{f}=\sqrt{\left|Z_{0,0}^{R}(f)\right|}$. Then $L_{m+2 l, m}^{R}(f)$ is invariant to scaling for any non-negative integer $m$ and $l$.

The proof is given in Appendix A.

Corollary 1. Let $f^{\prime}$ be a rotated version of $f$ with rotation angle $\alpha$. It holds for any non-negative integers $n$ and $m$ that

$$
L_{n, m}^{R}\left(f^{\prime}\right)=L_{n, m}^{R}(f) e^{-\mu m \alpha} .
$$

The proof of Corollary 1 is very similar to (21) and is thus omitted.

Then, using Corollary 1, we have

\section{Corollary 2. Let}

$$
\varphi_{n, k}^{m}(f)=L_{n, m}^{R}(f)\left(L_{k, m}^{R}(f)\right)^{*}, m \leq n, m \leq k, n-m \text { and } k-m \text { being even. }
$$

Then, $\varphi_{n, k}^{m}(f)$ is invariant to both image rotation and scaling for any non-negative integer $n, k$ and $m$.

The proof of Corollary 2 is very similar to that of Theorem 1 and is also omitted.

Combining (20) and (33), the RST invariants of QZMs (QZMIs) can be obtained as follows

Corollary 3. Let

$$
\bar{\varphi}_{n, k}^{m}(f)=\bar{L}_{n, m}^{R}(f)\left(\bar{L}_{k, m}^{R}(f)\right)^{*}, m \leq n, m \leq k, n-m \text { and } k-m \text { being even. }
$$

where $\bar{L}_{n, m}^{R}(f)$ is the scaling invariants defined in (31) using the central QZMs $\bar{Z}_{m+2 t, m}^{R}(f)$ instead of $Z_{m+2 t, m}^{R}(f)$. Then, $\bar{\varphi}_{n, k}^{m}(f)$ is invariant to image RST transformation for any non-negative integer $n, k$ and $m$.

Remark 1. By using the symmetric property of $R_{n, m}(r)$ with $m$, it can be easily proven that Corollary 3 is also true for $\mathrm{m}<0$.

\section{Experimental results}

In this section, several experiments are carried out to test the invariance property of the proposed descriptors to various geometric transformations, and their robustness to different kinds of noises. Note that the Zernike moments are calculated with the modified Kintner's method [36] and images are mapped inside the unit circle before the moment computation. 


\section{A. Test of color image reconstruction capability}

For this experiment, the standard Lena and Pepper images of size $256 \times 256$ were used. The reconstructed images using (9) with different values of $M$ are shown in Fig. 1 . Let $f(x, y)$ be the original image and $\hat{f}(x, y)$ be the reconstructed image, then the following normalized mean square error $\varepsilon^{2}$ [17] is used to measure the accuracy of the reconstructed images

$$
\varepsilon^{2}=\frac{\sum_{x=1}^{N} \sum_{y=1}^{N}|f(x, y)-\hat{f}(x, y)|^{2}}{\sum_{x=1}^{N} \sum_{y=1}^{N}|f(x, y)|^{2}} .
$$

The reconstruction errors are also given in Fig. 1. The results show that the reconstructed images are very close to the original image.

\section{B. Test of invariance to RST transformations}

For the experiments presented in this subsection as well as in the subsection a), a set of thirteen images (Fig. 2) with size $96 \times 72$ has been chosen from the public Amsterdam Library of Object Images (ALOI) database [37]. In order to contain the entire transformed image after transformation, the actual size of all the original images is $152 \times 128$ by adding some background pixels.

To test the invariance of the proposed QZMIs with respect to RST transformations, obj1 (Fig. 2(a)) was undergone different geometric transformations (Fig. 3). The proposed QZMIs defined in (34) of order from 1 to 4 were calculated for each image (the invariant of order zero is used to achieve the scale invariance in all the methods based on Zernike moments). Table 1 shows the modulus of invariant values and that of $\sigma \mu$, where $\mu$ denotes the mean of invariants and $\sigma$ the standard deviation. It can be seen from this table that excellent results have been obtained whatever the geometric transformations $(\sigma / \mu \leq 1.369 \%)$.

\section{Color object recognition}

To further assess the performance of the proposed QZMIs to RST transformation and their robustness against noise, three object recognition procedures were conducted.

a) Performance comparison of QZMIs and Zernike moment invariants based on RGB decomposition and graying in terms of recognition efficiency

By proceeding in a similar way as that described in section 3 for color image, we can also derive the Zernike moment invariants with respect to RST transformation for single channel of the RGB image and the gray-scale image obtained with image graying. In both cases, the conventional Zernike moments are concerned. So, for image graying, a set of Zernike moment invariants denoted by GZMIs are extracted from the graying image of the RGB image; for RGB decomposition, those invariants for three channels are grouped in a whole set (denoted hereafter by DZMIs). In order to compare the proposed QZMIs with GZMIs and DZMIs in terms of recognition efficiency, we use almost the same number of invariants in the experiment: The GZMIs include 19 non-zero real-valued invariants with order from 2 to 6 (the invariants of order one equal zero for gray-scale image when the central moments are concerned, but this is not the case for both DZMIs and QZMIs). The DZMIs contain 18 real-valued invariants with order from 1 to 3. QZMIs include 20 real-valued numbers with order from 1 to 4 .

We used thirteen images shown in Fig. 2 as the training set. To obtain the testing set, each image was translated with $\Delta x=$ $8, \Delta y=5$ (other translation can also be chosen), rotated with angle $\alpha \in\{0,30, \ldots, 300,330\}$, and scaled with scaling factor $\lambda$ $\in\{0.5,0.75, \ldots, 1.75,2.0\}$, forming a set of 1092 images, and a bilinear interpolation was used when required. This was followed by adding a white Gaussian noise with different standard deviations and a salt-and-pepper noise with different noise densities. Fig. 4 shows some examples of the transformed and corrupted images.

These three moment invariant sets are normalized according to the method presented by Suk and Flusser [7]

$$
I_{p}=\operatorname{sign}\left(I_{p}\right)\left|I_{p}\right|^{1 / r}, p=1,2, \ldots, n \text {. }
$$

where $r$ is the number of moments in one term and $n$ is the size of moment invariant set. Then, the minimum-Euclideandistance is used as the classifier.

The recognition results of three different invariant descriptors for both noise-free and noisy cases are shown in Table 2. The results show that: (1) the recognition results are quite good (100\%) for each method in noise-free case; (2) the recognition rates decrease with the noise level going up. However, the proposed QZMIs perform much better than other types of invariants whatever the noise and the noise level. Although some images are highly corrupted, the recognition rates of 
QZMIs are still better than 92\%. However, this is not the case for the other two methods; (3) the worst is GZMIs. The reason is that graying loses some color information, which may be important in object recognition.

b) Performance comparison of the new rotation invariants and the Zernike moment rotation invariants presented in [31]

To compare the new rotation invariants defined in (23) (denoted by QZMRIs) and the rotation invariants presented in our previous work [31] (denoted by QZMRIs ${ }_{I C P R}$ ), fourteen images with size $128 \times 128$ (Fig. 5) selected from the public Columbia Object Image Library (COIL-100) database [38] were used as the training set. The actual size of all the original images is $204 \times 204$. Then, each image was rotated with angle varying from $0^{\circ}$ to $350^{\circ}$ every $5^{\circ}$, forming a testing set of 1008 images. Moreover, these testing images were corrupted by a white Gaussian noise and a salt-and-pepper noise, respectively. A set of 20 QZMRIs $_{\text {ICPR }}$, whose order from 0 to 7, were used for object recognition. We used two sets of QZMRIs. The first one (denoted by QZMRIs ${ }_{21}$ ) includes 21 real-valued invariants of order from 0 to 4 . The second one (denoted by QZMRIs 56 )

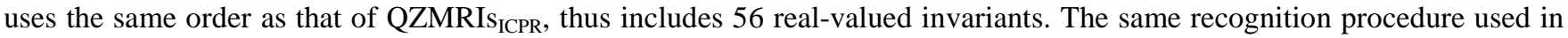
subsection a) is adopted.

Table 3 shows the recognition rates of these methods. It can be seen that the proposed new invariant descriptors have

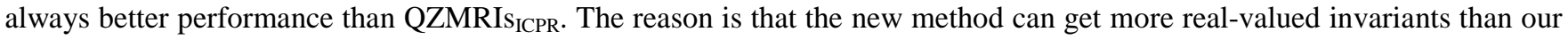
previous method when the same order of moments is used. When the number of moment invariants used in both methods are nearly the same, the new descriptors require lower order of moments, thus they are less sensitive to noise.

c) Performance comparison of the QZMIs and the existing moment invariants

In order to compare our QZMIs with the geometric/photometric invariants (GPIs) reported in [5] which are invariant to affine geometric deformations and diagonal photometric transformations invariants, the affine color moment invariants (ACMIs) proposed in [7] and QFMM invariants (QFMMIs) presented in [32], the nearly same number of invariants is used in all the methods: The 21 GPIs are the invariants described in Table 1 of Ref. [5]; The 21 ACMIs are the invariants used in [7]; The 24 QFMMIs are the invariants shown in (15) of Ref. [32] with order from 0 to 4 except the invariant of order 2 with repetition 0 which is used to achieve the scaling invariance. Our 20 QZMIs are the same as those used in subsection a).

We also used the fourteen images shown in Fig. 5 as the training set. To obtain the testing set, each image was transformed with the same transformations as those presented in subsection a) except for the translation (the actual translation is $\Delta x=-11, \Delta y=9$ ), forming a set of 1176 images. Then, to test the robustness against noise, these testing images were added a white Gaussian noise and a salt-and-pepper noise, respectively. The minimum-Euclidean-distance is used as classifier after the normalization with (36) for the four types of invariants.

The recognition results using different moment invariants are summarized in Table 4. It can be observed from this table that: (1) the invariants based on quaternion moments (QZMIs and QFMMIs) are more robust against noise than those based on conventional moments (GPIs and ACMIs); (2) the invariants based on orthogonal Zernike moments (QZMIs) are better than those based on non-orthogonal moments (QFMMIs) in noisy case. It is in accordance with that reported in [19], where the authors pointed out that the orthogonal Zernike moments have on overall better performance than non-orthogonal ones.

\section{Template matching}

The objective of the last experiment is to verify the performance of our descriptors for real outdoor scene images after RST transformation. The template matching test was performed. For that purpose, two pictures (Fig. 6) were taken by digital camera (Panasonic DMC-FZ50) with different focus and varying position through a rotation of the camera. Then, nine circular areas with radius $r=11$ in Fig. 6(a) were extracted as templates (numbered from 1 to 9). The scale factor between two images is obtained with the automatic scale selection [39]. Then, the scaled template was shifted across the other image (Fig. 6(b)) and in each position the normalized invariants were calculated and compared with the invariants of the original templates. For more detail about the matching procedure, we refer to [40]. The 'matched position' we consider corresponds to the location where the Euclidean distance $d(f, g)$ reaches its minimum value, with $f$ representing the template of the original image and $g$ the template of the transformed image. The matching results obtained by different moment invariants are shown in Fig. 6(c), (d), (e) and (f). It can be observed form these figures that our QZMIs can match correctly for eight templates with a reasonable error (within one pixel), but the number of correctly matched templates is only three for GPIs (number 2,5 and 9) and for ACMIs (number 3, 4 and 9), five for QFMMIs (number 4, 6, 7, 8 and 9). These results further validate the effectiveness of the proposed QZMIs.

\section{Conclusion}

In this paper, we have extended the conventional Zernike moment defined in gray-scale image to color image using the algebra of quaternions. The moment invariants with respect to RST transformations have been constructed. The advantages of the proposed QZMs and QZMIs over the existing descriptors are as follows: (1) the proposed QZMs are based on the 
orthogonal Zernike polynomials, thus they are more robust to noise; (2) the quaternion-type representation, treating a color image as a vector field, is used in the definition of moments; (3) the constructed QZMIs are quaternion-valued invariants instead of real-valued invariants, thus they provide more real-valued invariants. The results obtained from both simulation and real data show that the proposed descriptors are more robust to noise and have more discriminative power than the existing methods.

\section{Acknowledgement}

The authors would like to thank the anonymous reviewers and Associate Editor for their insightful suggestions which helped improve the manuscript. This work was supported by the National Basic Research Program of China under Grant 2011CB707904, the NSFC under Grants 61073138, 60911130370, and 60972163, the Ningbo Natural Science Foundation under Grants 2010A610106 and 2009A610090, and the Natural Science Foundation of Jiangsu Province under Grants SBK200910055 and BK2010426.

\section{Appendix A}

Proof of Theorem 2. Equation (31) can be written in matrix form as

$$
\left(\begin{array}{c}
Z_{m, m}^{R}\left(f^{\prime \prime}\right) \\
Z_{m+2, m}^{R}\left(f^{\prime \prime}\right) \\
\vdots \\
Z_{m+2 l, m}^{R}\left(f^{\prime \prime}\right)
\end{array}\right)=C_{l}^{m} \operatorname{diag}\left(\lambda^{m+2}, \lambda^{m+4}, \cdots, \lambda^{m+2 l+2}\right) D_{l}^{m}\left(\begin{array}{c}
Z_{m, m}^{R}(f) \\
Z_{m+2, m}^{R}(f) \\
\vdots \\
Z_{m+2 l, m}^{R}(f)
\end{array}\right) .
$$

Applying (32) to the transformed image $f^{\prime \prime}$, it can also be written in matrix form as

$$
\left(\begin{array}{c}
L_{m, m}^{R}\left(f^{\prime \prime}\right) \\
L_{m+2, m}^{R}\left(f^{\prime \prime}\right) \\
\quad \vdots \\
L_{m+2 l, m}^{R}\left(f^{\prime \prime}\right)
\end{array}\right)=C_{l}^{m} \operatorname{diag}\left(\Gamma_{f^{\prime \prime}}^{-(m+2)}, \Gamma_{f^{\prime \prime}}^{-(m+4)}, \cdots, \Gamma_{f^{\prime}}^{-(m+2 l+2)}\right) D_{l}^{m}\left(\begin{array}{c}
Z_{m, m}^{R}\left(f^{\prime \prime}\right) \\
Z_{m+2, m}^{R}\left(f^{\prime \prime}\right) \\
\vdots \\
Z_{m+2 l, m}^{R}\left(f^{\prime \prime}\right)
\end{array}\right) .
$$

Based on the definition of $\Gamma_{f}$, it can be easily verified that

$$
\Gamma_{f^{\prime \prime}}=\lambda \Gamma_{f} \text {. }
$$

Substituting (A1) and (A3) into (A2), and using the identity $D_{l}^{m} C_{l}^{m}=I$, where $I$ is the identity matrix, we obtain

$$
\begin{aligned}
\left(\begin{array}{c}
L_{m, m}^{R}\left(f^{\prime \prime}\right) \\
L_{m+2, m}^{R}\left(f^{\prime \prime}\right) \\
\vdots \\
L_{m+2 l, m}^{R}\left(f^{\prime \prime}\right)
\end{array}\right) & =C_{l}^{m} \operatorname{diag}\left(\Gamma_{f}^{-(m+2)}, \Gamma_{f}^{-(m+4)}, \cdots, \Gamma_{f}^{-(m+2 l+2)}\right) \\
& \times \operatorname{diag}\left(\lambda^{-(m+2)}, \lambda^{-(m+4)}, \cdots, \lambda^{-(m+2 l+2)}\right) D_{l}^{m} C_{l}^{m} \\
& \times \operatorname{diag}\left(\lambda^{m+2}, \lambda^{m+4}, \cdots, \lambda^{m+2 l+2}\right) D_{l}^{m}\left(\begin{array}{c}
Z_{m, m}^{R}(f) \\
Z_{m+2, m}^{R}(f) \\
\vdots \\
Z_{m+2 l, m}^{R}(f)
\end{array}\right) \\
= & C_{l}^{m} \operatorname{diag}\left(\Gamma_{f}^{-(m+2)}, \Gamma_{f}^{-(m+4)}, \cdots, \Gamma_{f}^{-(m+2 l+2)}\right) D_{l}^{m}\left(\begin{array}{c}
Z_{m, m}^{R}(f) \\
Z_{m+2, m}^{R}(f) \\
\vdots \\
Z_{m+2 l, m}^{R}(f)
\end{array}\right)=\left(\begin{array}{c}
L_{m, m}^{R}(f) \\
L_{m+2, m}^{R}(f) \\
\vdots \\
L_{m+2 l, m}^{R}(f)
\end{array}\right) .
\end{aligned}
$$

The proof has been completed.

\section{References}

[1] A. Khotanzad, and Y.H. Hong, Invariant image recognition by Zernike moments, IEEE Trans. Pattern Anal. Machine Intell. 12 (1990) $489-497$.

[2] F. Zhang, S.Q. Liu, D.B. Wang, and W. Guan, Aircraft recognition in infrared image using wavelet moment invariants, Image Vis. Comput. 27 (2008) 
313-318.

[3] Y.H. Lin, and C.H. Chen, Template matching using the parametric template vector with translation, rotation and scale invariance, Pattern Recognit. 41 (2008) 2413-2421.

[4] C.Y. Yang, and J.J. Chou, Classification of rotifers with machine vision by shape moment invariants, Aquac. Eng. 24 (2000) $33-57$.

[5] F. Mindru, T. Tuytelaars, L. V. Gool, and T. Moons, Moment invariants for recognition under changing viewpoint and illumination, Comput. Vis. Image Und. 94 (2004) 3-27.

[6] F. Mindru, T. Moons, and L. V. Gool, Color-based moment invariants for the viewpoint and illumination independent recognition of planar color patterns, In: Proc. Int. Conf. Advances in Pattern Recognition (ICAPR'98) (1998) 113-122.

[7] T. Suk, and J. Flusser, Affine moment invariants of color images, In: Proc. CAIP 2009, LNCS5702 (2009) $334-341$.

[8] Y.K. Chan, and C.C. Chang, A color image retrieval method based on color moment and color variance of adjacent pixels, Int. J. Pattern Recognit. Artif. Intell. 16 (2002) 113-125.

[9] F. Zernike, Beugungstheorie des Schneidenverfahrens und seiner verbesserten Form, der Phasenkontrastmethode (Diffraction theory of the cut procedure and its improved form, the phase contrast method), Physica 1 (1934) 689-704.

[10] M. Teague, Image analysis via the general theory of moments, J. Opt. Soc. Am. 70 (1980) 920-930.

[11] J. Wang, and G. Healey, Using Zernike moments for the illumination and geometry invariant classification of multispectral texture, IEEE Trans. Image Process. 7 (1998) 196-203.

[12] W.Y. Kim, and Y.S. Kim, A region-based shape descriptor using Zernike moments, Signal Process.: Image Commun. 16 (2000) 95-102.

[13] C. Kan, and M.D. Srinath, Invariant character recognition with Zernike and orthogonal Fourier-Mellin moments, Pattern Recognit. 35 (2002) 143-154.

[14] N. Kanaya, Y. Liguni, and H. Maeda, 2-D DOA estimation method using Zernike moments, Signal Process. 82 (2002) 521-526.

[15] H.S. Kim, and H.K. Lee, Invariant image watermark using Zernike moments, IEEE Trans. Circuits Systems for Video Technol. 13 (2003) $766-775$.

[16] M. Novotni, and R. Klein, Shape retrieval using 3D Zernike descriptors, Comput. Aided Design 36 (2004) 1047-1062.

[17] J. Revaud, and G. Lavoue, A. Baskurt, Improving Zernike moments comparison for optimal similarity and rotation angle retrieval, IEEE Trans. Pattern Anal. Mach. Intell. 31 (2009) 627-636.

[18] Z. Chen, and S.K. Sun, A Zernike moment phase-based descriptor for local image representation and matching, IEEE Trans. Image Process. 19 (2010) 205-219.

[19] C.H. Teh, and R.T. Chin, On image analysis by the method of moments, IEEE Trans. Pattern Anal. Machine Intell. 10 (1988) 496-513.

[20] H.Z. Shu, L.M. Luo, and J.L. Coatrieux, Moment-based approaches in image Part 1: basic features, IEEE Eng. Med. Biol. Mag. 26 (2007) 70-74.

[21] H.Z. Shu, L.M. Luo, and J.L. Coatrieux, Moment-based approaches in image Part 2: invariance, IEEE Eng. Med. Biol. Mag. 27 (2008) 81-83.

[22] T.A. Ell, and S.J. Sangwine, Hypercomplex Fourier transforms of color images, IEEE Trans. Image Process. 16 (2007) 22-35.

[23] T.A. Ell, Hypercomplex spectral transforms, Ph.D dissertation, Minneapolis: Minnesota University, 1992.

[24] S.J. Sangwine, Fourier transforms of colour images using quaternion or hypercomplex, numbers, Electron. Lett. 32 (1996) 1979-1980.

[25] C.E. Moxey, S.J. Sangwine, and T.A. Ell, Color-grayscale image registration using hypercomplex phase correlation, In: Proc. IEEE Int. Conf. Image Process. (ICIP 2002) 3(2002) 247-250.

[26] W. Feng, B. Hu, and C. Yang, A subpixel color image registration algorithm using quaternion phase-only correlation, In: Proc. IEEE Int. Conf. Audio, Language and Image Process. (ICALIP 2008) (2008) 1045-1049.

[27] P. Bas, N.L. Bihan, and J.M. Chassery, Color image watermarking using quaternion Fourier transform, In: Proc. IEEE Int. Conf. Acoustic, Speech, and Signal Process. (ICASSP 2003) 3(2003) 521-524.

[28] X.J. Ma, Y. Xu, L. Song, X.K. Yang, and H. Burkhardt, Color image watermarking using local quaternion Fourier spectral analysis, In: Proc. IEEE Int. Conf. Multimedia and Expo. (ICME 2008) (2008) 233-236.

[29] D.S. Alexiadis, and G.D. Sergiadis, Estimation of motions in color image sequences using hypercomplex Fourier transforms, IEEE Trans. Image Process. 18 (2009) 168-187.

[30] D. Assefa, L. Mansinha, K. F. Tiampo, H. Rasmussen, and K. Abdella, Local quaternion Fourier transform and color image texture analysis, Signal Process. 90 (2010) 1825-1835.

[31] B.J. Chen, H.Z. Shu, H. Zhang, G. Chen, and L.M. Luo, Color image analysis by quaternion Zernike moments, In: Proc. 2010 20th International Conference on Pattern Recognition (ICPR2010) (2010) 625-628.

[32] L.Q. Guo, and M. Zhu, Quaternion Fourier-Mellin moments for color image, Pattern Recognit. 44 (2011) 187-195.

[33] W. R. Hamilton, Elements of quaternions, Longmans Green, London, U.K., 1866.

[34] C. W. Chong, P. Raveendran, R. Mukundan. Translation invariants of Zernike moments, Pattern Recognit. 36(2003) $1765-1773$.

[35] H.Z. Shu, L.M. Luo, G.N. Han, and J.L. Coatrieux, A general method to derive the relationship between two sets of Zernike coefficients corresponding to different aperture sizes, J. Opt. Soc. Am. A 23 (2006) 1960-1966.

[36] C.W. Chong, P. Raveendran, and R. Mukundan, A comparative analysis of algorithm for fast computation of Zernike moments, Pattern Recognit. 36 (2003) 731-742.

[37] J.M. Geusebroek, G.J. Burghouts, and A.W.M. Smeulders, The Amsterdam library of object images, Int. J. Comput. Vis. 61 (2005) 103-112.

[38] S. A. Nene, S. K. Nayar, and H. Murase, Columbia Object Image Library (COIL-100), Technical Report CUCS-006-96 (1996).

[39] T. Lindeberg, Feature detection with automatic scale selection, Int. J. Comput. Vis. 30 (1998) $79-116$.

[40] J. Flusser, and T. Suk, Degraded image analysis: an invariant approach, IEEE Trans. Pattern Anal. Machine Intell. 20 (1998) $590-603$. 


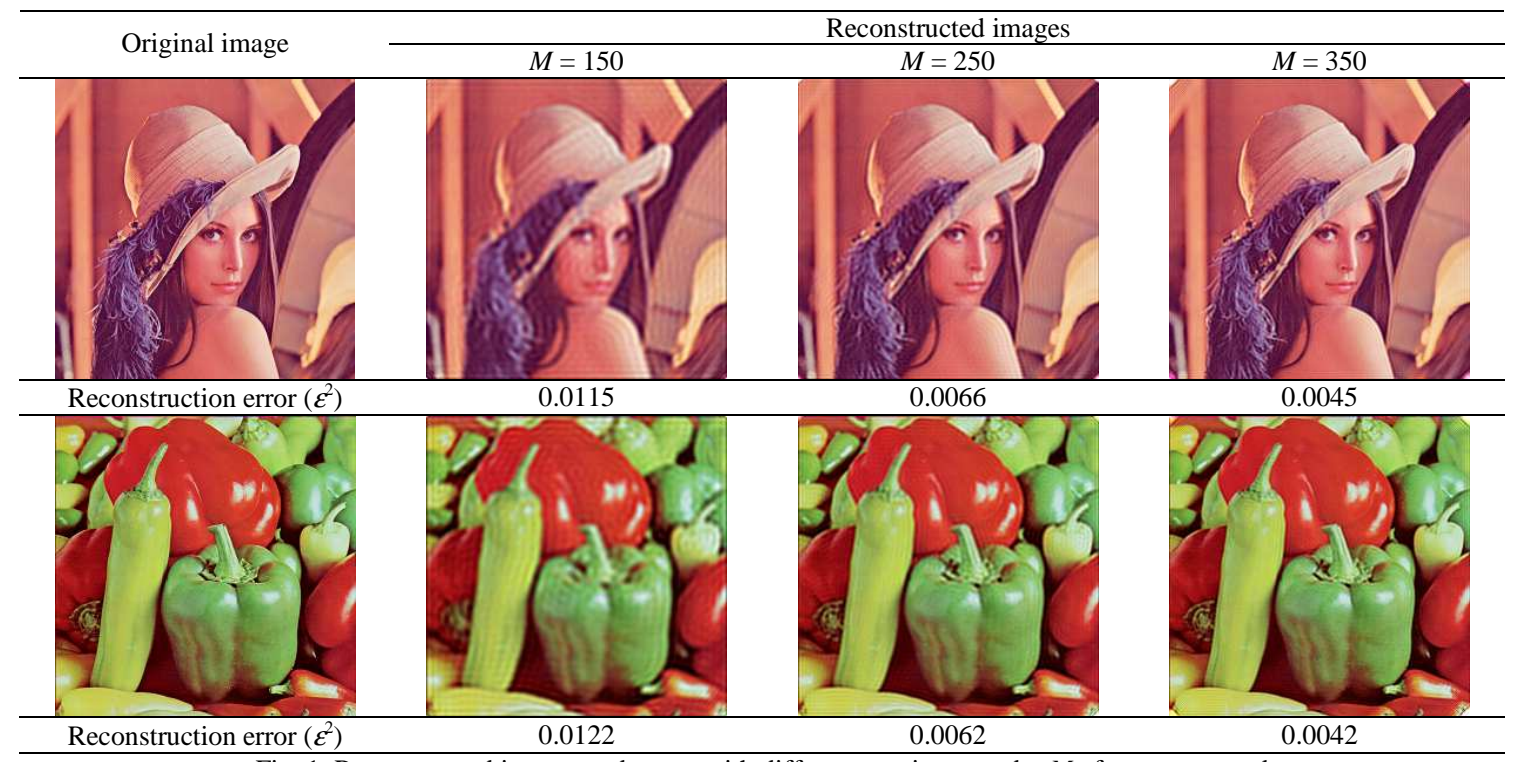

Fig. 1. Reconstructed images and errors with different maximum order $M$ of moments used

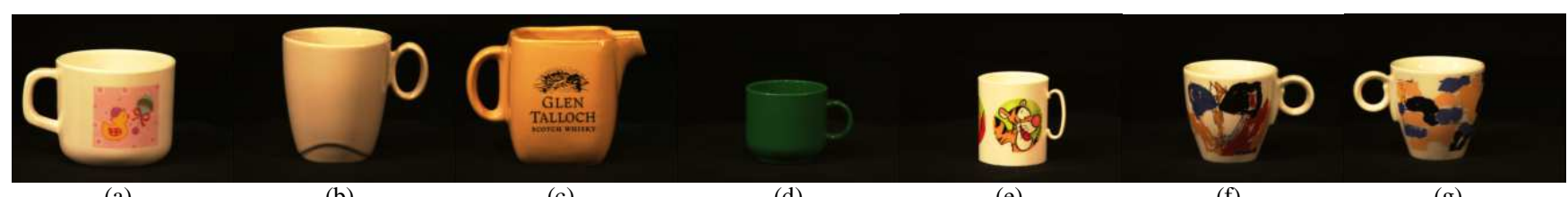

(a)

(b)

(c)

(d)

(e)

(f)

(g)

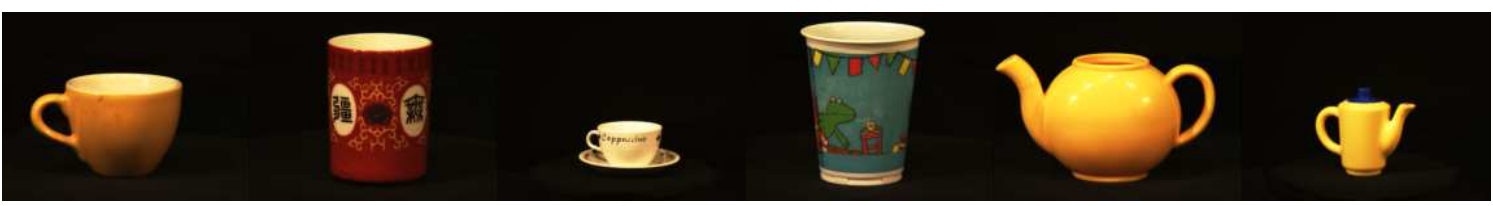

(h)

(i)

(j)

(k)

(1)

Fig. 2. Thirteen objects selected from the Amsterdam Library of Object Images database

(m)

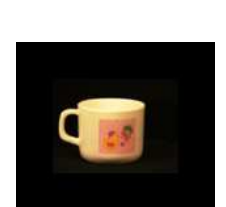

(a) original

image

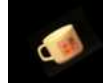

(b) $\Delta x=$

$8, \Delta y=$

$5, \lambda=$ $0.5, \theta=$

$30^{\circ}$

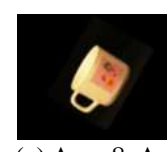

(c) $\Delta x=8, \Delta y$ $=5, \lambda=0.75$, $\theta=60^{\circ}$

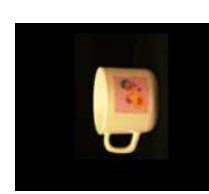

(d) $\Delta x=8, \Delta y=$ 5, $\lambda=1.0, \theta=$

$90^{\circ}$

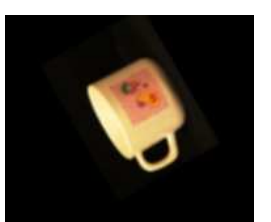

(e) $\Delta x=8, \Delta y=5$ $\lambda=1.25, \theta=120^{\circ}$

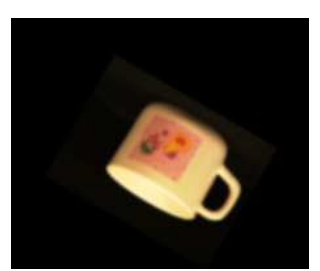

(f) $\Delta x=-5, \Delta y=-8, \lambda=$ $1.5, \theta=150^{\circ}$

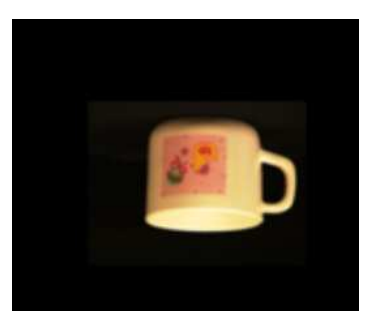

(g) $\Delta x=-5, \Delta y=-8, \lambda=1.75$, $\theta=180^{\circ}$

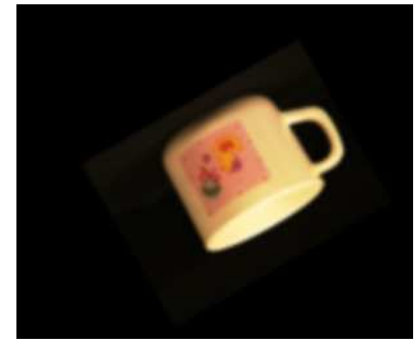

(h) $\Delta x=-5, \Delta y=-8, \lambda=2.0, \theta=$ $210^{\circ}$

Fig. 3. Geometric transformed images of Fig. 2(a) ( $\Delta x$ is the translation (in pixel) along the $x-$ axis, $\Delta y$ the translation along the y-axis, $\lambda$ the scaling factor, $\theta$ the rotation angle) 


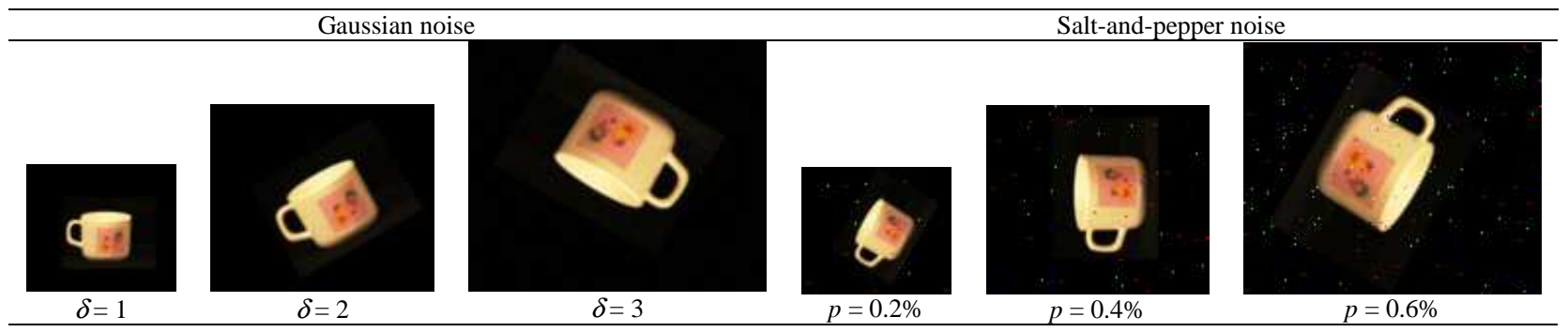

Fig. 4. Examples of image with different geometric transformations and varying noise ( $\delta$ is the STD of Gaussian noise, $p$ is the density of saltand-pepper noise)
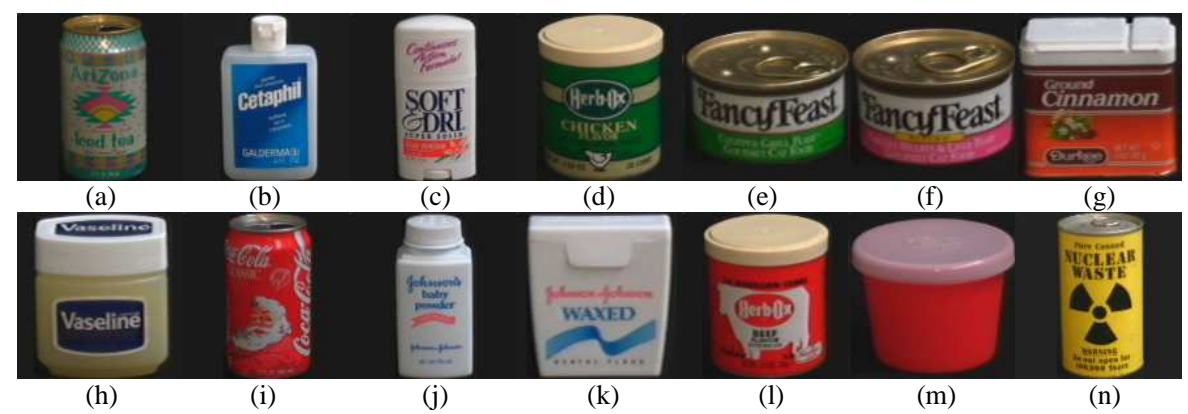

Fig. 5. Fourteen objects selected from the Columbia University Image Library database

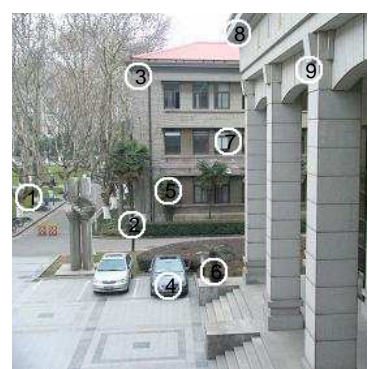

(a)

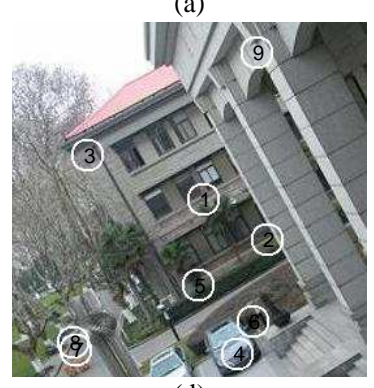

(d)

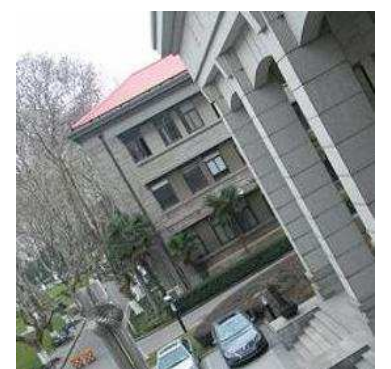

(b)

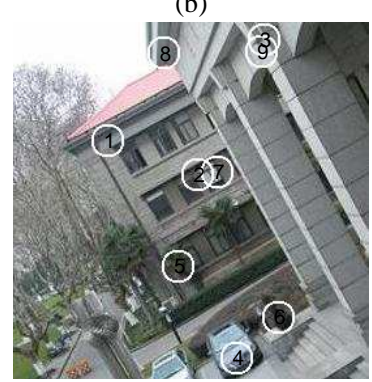

(e)

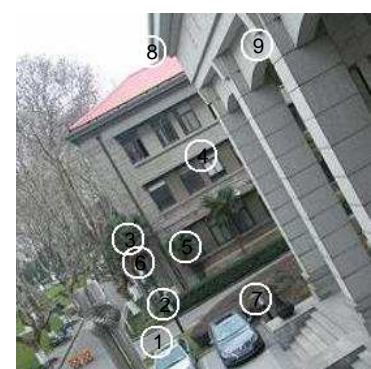

(c)

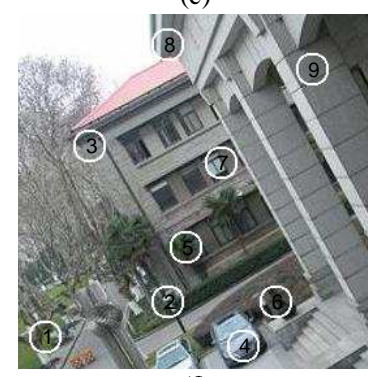

(f)

Fig. 6. Images of the outdoor scene. (a) and (b) are the original image and the transformed image, (c), (d), (e) and (f) are the matched templates using GPIs, ACMIs, QFMMIs and QZMIs, respectively 
Table 1. Modulus of QZMIs of images shown in Fig. 3

\begin{tabular}{cccccccccc}
\hline & Fig.3(a) & Fig.3(b) & Fig.3(c) & Fig.3(d) & Fig.3(e) & Fig.3(f) & Fig.3(g) & Fig.3(h) & $\sigma / \mu(\%)$ \\
\hline$\left|\bar{\varphi}_{1,1}^{1}\right|$ & $1.294 \mathrm{e}-05$ & $1.297 \mathrm{e}-05$ & $1.287 \mathrm{e}-05$ & $1.294 \mathrm{e}-05$ & $1.294 \mathrm{e}-05$ & $1.293 \mathrm{e}-05$ & $1.292 \mathrm{e}-05$ & $1.291 \mathrm{e}-05$ & 0.226 \\
\hline$\left|\bar{\varphi}_{2,0}^{0}\right|$ & 5.174 & 5.174 & 5.174 & 5.174 & 5.174 & 5.174 & 5.174 & 5.174 & 0.00 \\
\hline$\left|\bar{\varphi}_{2,2}^{2}\right|$ & $1.590 \mathrm{e}-06$ & $1.589 \mathrm{e}-06$ & $1.592 \mathrm{e}-06$ & $1.590 \mathrm{e}-06$ & $1.563 \mathrm{e}-06$ & $1.589 \mathrm{e}-06$ & $1.594 \mathrm{e}-06$ & $1.591 \mathrm{e}-06$ & 0.626 \\
\hline$\left|\bar{\varphi}_{3,1}^{1}\right|$ & $5.142 \mathrm{e}-05$ & $5.155 \mathrm{e}-05$ & $5.114 \mathrm{e}-05$ & $5.142 \mathrm{e}-05$ & $5.141 \mathrm{e}-05$ & $5.140 \mathrm{e}-05$ & $5.134 \mathrm{e}-05$ & $5.132 \mathrm{e}-05$ & 0.228 \\
\hline$\left|\bar{\varphi}_{3,3}^{3}\right|$ & $9.627 \mathrm{e}-10$ & $9.735 \mathrm{e}-10$ & $9.545 \mathrm{e}-10$ & $9.627 \mathrm{e}-10$ & $9.290 \mathrm{e}-10$ & $9.650 \mathrm{e}-10$ & $9.619 \mathrm{e}-10$ & $9.610 \mathrm{e}-10$ & 1.369 \\
\hline$\left|\bar{\varphi}_{4,0}^{0}\right|$ & 8.552 & 8.552 & 8.552 & 8.552 & 8.552 & 8.552 & 8.552 & 8.552 & 0.00 \\
\hline$\left|\bar{\varphi}_{4,2}^{2}\right|$ & $7.884 \mathrm{e}-06$ & $7.875 \mathrm{e}-06$ & $7.894 \mathrm{e}-06$ & $7.884 \mathrm{e}-06$ & $7.747 \mathrm{e}-06$ & $7.877 \mathrm{e}-06$ & $7.903 \mathrm{e}-06$ & $7.886 \mathrm{e}-06$ & 0.635 \\
\hline$\left|\bar{\varphi}_{4,4}^{4}\right|$ & $7.513 \mathrm{e}-11$ & $7.524 \mathrm{e}-11$ & $7.540 \mathrm{e}-11$ & $7.513 \mathrm{e}-11$ & $7.569 \mathrm{e}-11$ & $7.535 \mathrm{e}-11$ & $7.524 \mathrm{e}-11$ & $7.504 \mathrm{e}-11$ & 0.272 \\
\hline
\end{tabular}

Table 2. Recognition rates (\%) of different Zernike moment invariants in object recognition with RST transformation and noise

\begin{tabular}{cccc}
\hline & GZMIs & DZMIs & QZMIs \\
\hline Noise-free & 100.00 & 100.00 & 100.00 \\
\hline Gaussian noise with STD $=1$ & 69.05 & 100.00 & 100.00 \\
\hline Gaussian noise with STD $=2$ & 48.08 & 90.66 & 92.31 \\
\hline Gaussian noise with STD $=3$ & 38.46 & 75.27 & 92.03 \\
\hline Salt-and-pepper noise with noise density $=0.2 \%$ & 83.97 & 94.05 & 96.61 \\
\hline Salt-and-pepper noise with noise density $=0.4 \%$ & 52.47 & 84.62 & 94.87 \\
\hline Salt-and-pepper noise with noise density $=0.6 \%$ & 44.51 & 77.47 & 92.86 \\
\hline Average rate & 62.36 & 88.87 & 95.53 \\
\hline
\end{tabular}

Table 3. Recognition rates (\%) of different Zernike moment invariants in object recognition with rotation and noise

\begin{tabular}{cccc}
\hline & QZMRIS $_{\text {ICPR }}$ & QZMRIs $_{21}$ & QZMRIs $_{56}$ \\
\hline Noise-free & 100.00 & 100.00 & 100.00 \\
\hline Gaussian noise with STD = 40 & 90.28 & 99.80 & 99.90 \\
\hline Gaussian noise with STD $=50$ & 73.41 & 98.71 & 99.21 \\
\hline Gaussian noise with STD = 60 & 51.49 & 95.54 & 97.32 \\
\hline Salt-and-pepper noise with noise density = 5\% & 98.91 & 100.00 & 100.00 \\
\hline Salt-and-pepper noise with noise density = 10\% & 72.02 & 96.53 & 96.83 \\
\hline Salt-and-pepper noise with noise density $=15 \%$ & 49.90 & 89.19 & 90.67 \\
\hline Average rate & 76.57 & 97.11 & 97.70 \\
\hline
\end{tabular}

Table 4. Recognition rates (\%) of different moment invariants in object recognition with RST transformation and noise

\begin{tabular}{ccccc}
\hline & GPIs & ACMIs & QFMMIs & QZMIs \\
\hline Noise-free & 100.00 & 100.00 & 100.00 & 100.00 \\
\hline Gaussian noise with STD = 5 & 86.22 & 99.91 & 100.00 & 100.00 \\
\hline Gaussian noise with STD = 7 & 64.71 & 92.52 & 96.60 & 100.00 \\
\hline Gaussian noise with STD = 9 & 50.00 & 87.16 & 89.63 & 96.26 \\
\hline Salt-and-pepper noise with noise density $=1 \%$ & 94.56 & 97.70 & 98.64 & 99.91 \\
\hline Salt-and-pepper noise with noise density = 2\% & 84.10 & 86.56 & 88.95 & 92.77 \\
\hline Salt-and-pepper noise with noise density = 3\% & 68.54 & 72.79 & 75.85 & 84.35 \\
\hline Average rate & 78.30 & 90.95 & 92.81 & 96.18 \\
\hline
\end{tabular}

